\title{
What studying worms, flies and fish says about autism
}

\section{BY SARAH DEWEERDT}

8 SEPTEMBER 2021

Illustration by Natalie Nelson

Listen to this story:

In a windowless room near the western edge of Vancouver, Canada, a team of researchers trains microscopes on dishes of tiny worms about the size of a comma in 12-point font. Some of the worms squirm atop a sticky, jelly-like platform of nutrients that is saltier in some places than in others. Others endure vibrations as a machine periodically taps the sides of their dishes.

Typical roundworms of this species, known scientifically as Caenorhabditis elegans, move toward salt - a sign of food - and crawl backward when they sense reverberations. But these roundworms crawl away from salt and often seem to ignore the knocks on their dishes. The strange behavior suggests that a mutation the worms have - in a top autism gene called PTEN — is harmful. "It might seem a little funny at first to study, say, tapping a dish with worms in it," says Troy McDiarmid, a former graduate student in the lab of Catharine Rankin, a behavioral neuroscientist at the University of British Columbia in Canada. "But that ends up being a really good readout" of the role autism-linked genes play in learning and behavior.

Most animal research on autism involves mice. As fellow mammals, mice are relatively closely related to people, but there's still debate about whether the behaviors of autism mouse models accurately mirror those of autistic people. And yet, in the past 20 years, some autism researchers have turned to even simpler animals, such as roundworms, fruit flies and zebrafish, for their investigations. In 2000, just one published autism 
study used zebrafish or roundworms, and four focused on fruit flies. In 2020, those numbers had risen to 29 studies for zebrafish, 30 for fruit flies and 8 for roundworms.

So how could a fish or a fly, let alone a roundworm, be the source of insights into a condition that involves language and complex cognitive skills? Autism researchers who work with these animals are careful not to overhype their possibilities. Their nervous systems are small and lack complex brain structures, and their genes are regulated differently from those in mammals. Yet the basic mechanisms by which cells function have a remarkable continuity across animals, as do the structure and function of many genes. Seemingly simple animals also may show complex behaviors: Flies have social networks; fish swim in sync with others of their kind; worms pick up on chemical signals from other worms to help them find food.

The major value of these model organisms is practical: They are relatively cheap and easy to maintain, with short generation times, and straightforward to manipulate genetically. So researchers can use them for rapid-fire testing of drugs or to get a quick read on the effects of mutations, as well as for exploratory work that would be too costly and time-consuming in mice. "You can do a lot of wonderful things in C. elegans," Rankin says.

These animals have helped link new genes to autism, sort out harmful from benign mutations, show how multiple autism-linked genes interact, and uncover neural circuits involved in sensory and social function. "Working on simple critters is an essential part of the information pipeline," says James Rand, a retired molecular biologist who worked at the Oklahoma Medical Research Foundation in Oklahoma City.

\section{A worm of one's own:}

\section{Listen to this story:}

Rand began using roundworms to study nervous system development in the 1970s. In the mid-2000s, when some of the first studies of autism genetics were published, he remembered something his wife had brought up years before, around the time their two sons were diagnosed with autism. "Why don't you do something useful — study autism and so on?" she had challenged 


\section{Spectrum | Autism Research News}

https://www.spectrumnews.org

him. Rand had said that he would, as soon as he saw an opportunity.

Now that opportunity had arrived. The studies implicated a family of genes for proteins called neuroligins, or NLGN, in autism. People have four NLGN genes, but roundworms have just one.

Read more: Getting eight arms around autism

Rand obtained a strain of worms that had a mutation in that gene. Then he applied what he calls a "standard $C$. elegans paradigm" to investigate its effects, documenting gene expression, survival and behavior in the mutant worms in response to various challenges.

Worms with NLGN mutations have sensory-processing abnormalities, Rand and his team reported in 2010: They don't crawl away from a chemical that repels wildtype worms. They also succumb more readily to toxic metals and chemicals in their environment. Reviewers were skeptical that studying worms could say anything useful about autism. "Getting that paper accepted was a serious struggle," Rand recalls. But the results showed how roundworms might be used to investigate the impact of mutations in autism-linked genes.

And as the trickle of autism genetic data turned into a river in the following years, the need to get a rapid read on gene function increased. "We've come very far in being able to identify genes that are strongly associated with autism," says Ellen Hoffman, a psychiatric geneticist at Yale University who works with zebrafish. "But there's really a big gap between being able to identify these genes and understanding how the disruption of these genes actually leads to the clinical presentation of autism." Roundworms, flies and zebrafish can bridge that gap.

Technology has played a critical role as well. The advent of the CRISPR-Cas9 gene-editing system "enabled a lot more precise modeling of genetic variation," says McDiarmid, now a postdoctoral researcher in Jay Shendure's lab at the University of Washington in Seattle. Researchers could now recreate in an animal model, and thereby study, the exact mutations seen in people with autism. Roughly half of all human genes and 70 to 80 percent of genes implicated in autism have an equivalent version, or homolog, in roundworms. Rankin's team has used the tool to engineer specific versions of PTEN and other human genes in worms. (PTEN is so similar to the worm gene 


\section{Spectrum | Autism Research News}

https://www.spectrumnews.org

DAF18 that the human gene can replace the worm one with no harm to the animal.) In a study published last year, the researchers used the worms to analyze the effects of 19 PTEN variants found in autistic people.

And instead of manually documenting the behavior of one worm at a time, Rankin's team uses cameras to record the behavior of large groups of worms and a computer program to analyze the resulting images. Software called the Multi-Worm Tracker enables them to track 40 to 100 worms from a given strain simultaneously, capturing data on more than two dozen parameters, including a worm's shape, speed and crawling direction. The software greatly sped up the research, spitting out its analysis of a strain in just 15 minutes. "Before that, it would take two or three weeks of constant work," Rankin says.

"Working on simple critters is an essential part of the information pipeline." James Rand

In a 2020 study, Rankin and her team analyzed more than 27,000 worms representing 135 strains with mutations in the worm homologs of 98 autism-linked genes. Many of the worms show deficits in habituation, a process by which the brain tamps down its response to unchanging stimuli. It's a way of tuning out irrelevant input, and it is also disrupted in many people with autism. The results suggest that many autism-linked genes have common functions.

In a similar set of experiments, researchers engineered 20 de novo, or spontaneous, mutations seen in autistic people in the worm versions of 11 autism-linked genes. They assessed body shape, size, movement and reproduction in the mutant worms and found mostly subtle changes that suggest the mutations dampen but do not completely eliminate gene function. Next, the researchers plan to engineer worms with multiple variants found in the same autistic person, to learn how combinations of genes might lead to autism. "The worms are really good for making multiple genetic changes at the same time" because of their fast generation times, says lead researcher Paul Sternberg, a molecular geneticist at the California Institute of Technology in Pasadena.

\section{Fly society:}

\section{Listen to this story:}


In 2012, geneticist Santhosh Girirajan boarded a plane in Seattle, where he had just finished a postdoctoral fellowship at the University of Washington. On the plane, he cracked open a book he had received as a going-away present: "Time, Love, Memory," the story of biologist Seymour Benzer's Nobel Prize-winning discoveries about sleep and circadian rhythms in fruit flies.

By the time Girirajan landed in State College, Pennsylvania, where he was about to take a position at Pennsylvania State University, he had devoured half the book and was a fruit fly convert. Girirajan began to think about how fruit flies, Drosophila melanogaster, might aid his genetic studies of autism. In particular, he thought they might speed insights into how copy number variations (CNVs) - deletions or duplications of large stretches of DNA - contribute to the condition. Girirajan scrapped his plans to work with mice and set up a fly lab instead.

Because CNVs can contain more than two dozen genes, it can be tricky to figure out how the various genes contribute to autism traits. In the fly, it is possible to manipulate gene expression in specific tissues, such as the brain, eye or wing, enabling Girirajan to test the effects of these changes without killing the fly. Girirajan has turned down the expression of two genes at a time in the fly's eye and tracked the effects on eye development using a purpose-built computer program he calls Flynotyper. The program detects disruptions to the eye's structure by analyzing images of the eye, calculating the distances and angles between its parts. The effects on the eye serve as a proxy for those on the nervous system as a whole, because many genes affect the development of both, Girirajan says.

Using this method to analyze 565 pairs of genes revealed that interactions between many genes likely underlie the link between the CNV 16p11.2 and conditions such as autism. By contrast, a study of 3q29 involving 314 pairs of genes showed that a single gene called NCBP2 drives the developmental effects of the deletion. Girirajan is now conducting similar studies of other CNVs, such as $15 q 11.2,15 q 13.3$ and 1 q21.1.

Fruit flies are also advancing the understanding of sleep problems in autism, via both genetic manipulation and automated tracking of their behavior. In a study published this year, researchers tracked the sleep cycles of flies with mutations in the fly equivalent of the genes CHD8 and CHD7, which are associated with autism or autism traits, using a camera connected to software that records their movements throughout the day. The scientists found that the mutant flies wake up unusually often, reminiscent of the sleep difficulties seen in people with mutations in these genes.

By removing these genes from specific cell types, the researchers traced the problem to glial cells in the blood-brain barrier. The researchers then treated the problem by reducing the amount of time each day the flies spent in the dark to the minimum they need for sleep. Restricting the time spent in bed is a treatment for insomnia in people, and this similar intervention in flies consolidated their sleep into fewer, longer bouts, more like that of typical flies. "We really hope that our work is changing something about this perception that you cannot do anything" about sleep problems in 


\section{Spectrum | Autism Research News}

https://www.spectrumnews.org

children with autism, says lead researcher Annette Schenck of Radboud University Medical Center in Nijmegen, the Netherlands.

\section{Fishing expedition:}

\section{Listen to this story:}

Scientists have also studied the nighttime rituals of another autism model: zebrafish (Danio rerio). Gene-editing techniques enable scientists to create zebrafish with autism-linked mutations, and then they can easily assess how the mutations effect behavior. "We can take our fish larvae - at 5 days old they have this complex range of behaviors - we can easily pipette them into the wells of a 96-well plate, and then track different aspects of their locomotor activity," Hoffman says. The design offers the high throughput and easy replicability of a cell culture study, with the ability to gauge effects on animal behavior.

As a postdoctoral researcher, Hoffman tracked the behavior of 5-day-old zebrafish larvae lacking the autism-linked gene CNTNAP2 and found that they are hyperactive at night. The fish also have fewer-than-usual inhibitory neurons, which dampen neural activity, in the forebrain, replicating findings in mice lacking the same gene and adding heft to the relevance of the fish as an autism model.

Zebrafish are a useful tool for screening potential drugs for autism because chemical compounds can be added directly to the water the fish swim in. In their CNTNAP2 study, published in 2016, Hoffman and her colleagues tested the effects of 14 drugs on the larvae and showed that certain forms of the hormone estrogen can reverse the larvae's hyperactive behavior.

The animals are small and relatively inexpensive, so the team can use them to study the effects of many autism-linked genes in parallel. Hoffman's team is looking at brain activity, movement and sleep-wake cycles across multiple lines of zebrafish with mutations in the fish equivalents of 10 autism-linked genes, including CHD8, CNTNAP2, DYRK1A, GRIN2B and SCN2A. The researchers aim to identify shared characteristics across strains and to identify drugs that could reverse alterations in their behavior.

The CNTNAP2 zebrafish developed in Hoffman's lab are slated to appear on a list of validated zebrafish models curated by the Simons Foundation Autism Research Initiative (SFARI). (Spectrum is an editorially independent publication funded by SFARI.) The goal of that list is to make zebrafish research more reliable by steering researchers toward models that pass a test of 


\section{Spectrum | Autism Research News}

https://www.spectrumnews.org

genetic quality, says SFARI senior scientist Brigitta Gundersen.

Read more: Autism research makes the leap to frogs

Zebrafish larvae have another advantage: They are transparent for the first several days of life. As a result, researchers can see the larvae's internal organs, including the gut, enabling them to visualize the effects of autism mutations on gut function, which is often disrupted in autism. In the larvae, researchers can observe the rhythmic movements of the gut muscles, and food moving through the digestive system. "Things play out right in front of you," says Julia Dallman, associate professor of biology at the University of Miami in Coral Gables, Florida.

In studies published in 2019 and 2020, Dallman's team showed that the gut muscles contract, and food moves through the gut, unusually slowly in zebrafish with mutations in SYNGAP1 or SHANK3. In people, mutations in these genes are linked to both autism and gastrointestinal distress, including constipation and acid reflux. "When we initially set out to look at gut function in these models, my expectation was that [alterations] would be subtle," Dallman says. "It's not subtle at all." Her team's studies suggest that slightly different mechanisms underlie gut problems in the two fish strains, so treatment of constipation in autism may not be one size fits all. Dallman plans to test the effects of drugs on both gut function and behavior in the fish, to aid in the search for autism medications that don't worsen constipation, she says.

The transparency of zebrafish larvae also puts early brain development on display. Using specialized microscopes, researchers can visualize the activity of individual neurons and, because the fish are small, simultaneously track the activity of every neuron in the brain. "You're just observing the brain in an intact, alert, behaving, perceiving animal," says Ethan Scott, who studies sensory processing in zebrafish at the University of Queensland in Brisbane, Australia.

Zebrafish have a more similar brain structure to humans than do invertebrate models, Scott says. And although the fish lack a cerebral cortex, the structure at the surface of the human brain, they are useful for studying circuits in other parts of the brain. "It's all a matter of matching your question to the advantages and disadvantages of your model system," Scott says.

Scott is using zebrafish larvae with mutations in autism-linked genes to investigate alterations in sensory processing in autism. He and his colleagues are monitoring their brain activity in response to images shown on a computer screen, and to sounds of varying loudness. In a 2020 study, his 


\section{Spectrum | Autism Research News}

https://www.spectrumnews.org

team showed that zebrafish lacking FMR1, the gene mutated in fragile $\mathrm{X}$ syndrome, are hypersensitive to sound. And in four regions of the brain, their neurons react to sound with more frequent or stronger bursts of activity than those in typical fish. The work may help explain sensory hypersensitivity in autism, Scott says.

\section{Social creatures:}

\section{Listen to this story:}

Differences in social behavior are central to autism, and the roots of those behaviors extend throughout the animal kingdom, says Anne Simon, a behavioral genetecist at the University of Western Ontario in London, Canada. "Whether you're a fly or a human, you need to be able to recognize one from your species," she says. "You need to be able to go close enough but not too close." Animals need to group together to find food and mates, she explains, but they need space to avoid conflicts over resources.

Simon has established a way to test the effects of genes on social behavior in fruit flies. In a 2015 study, she and her colleagues found that these flies naturally engage in 'social distancing': In a triangle-shaped enclosure, they fly upward in an attempt to escape and eventually cluster near the apex of the triangle, but they keep a set amount of space between them. With this apparatus, she can probe the effects of autism-linked mutations on the flies' spacing, as a marker of social function. In 2015 and 2019, her team reported that flies with mutations in the Drosophila equivalents of NBEA and of FOXP1 and FOXP2 settle farther apart than typical flies do. Males that either lack or overexpress NLGN3 similarly spread out, as do female flies lacking NLGN3. But females that overexpress NLGN3 pack close together. The results suggest that these genes affect social brain circuits, Simon says.

"Whether you're a fly or a human, you need to be able to recognize one from your species." Anne Simon

In the wild, adult zebrafish swim in synchrony, or 'school,' a behavior that protects them from predators. Researchers are exploring how autism-linked genes affect schooling and other forms of 


\section{Spectrum | Autism Research News}

https://www.spectrumnews.org

fish fraternizing. They have found, for example, that zebrafish lacking SHANK3 show little interest in being near other fish; fish lacking oxytocin receptors opt for more alone time than other zebrafish do, and those lacking the gene RELN do not choose to interact with unfamiliar fish over familiar fish as is typical for the species. The RELN mutants lack the neurotransmitter serotonin in the hindbrain, the researchers found, and adding a drug to their water that boosts serotonin in the brain restores the preference for strangers.

"I was initially very skeptical" of using zebrafish to investigate the social fallout from mutations in autism genes, says Will Norton, a behavioral neuroscientist at the University of Leicester in the United Kingdom, who led the RELN study. A change in fish sociability is not necessarily indicative of a similar effect in people, he acknowledges, but the results in fish mirror those seen in RELN mouse models, so he thinks the fish social behavior is meaningful.

Zebrafish have other helpful social quirks. If two fish are separated by a transparent barrier, they tend to turn to face each other. Certain cells in the zebrafish forebrain that release the neurotransmitter acetylcholine seem to be necessary for this behavior: When these cells are genetically disabled, the fish spend less time oriented toward each other, according to a 2018 study. Mice have a similar cell population

Read more: Fish, frogs, flies and other fauna in scientific firsts

involved in social behavior in the forebrain, and such cells are likely to exist in people as well, says lead researcher Philip Washbourne, a neuroscientist at the University of Oregon in Eugene. "One thing that's lacking across all model organisms and in humans is just a really good understanding of the circuits involved in social behavior," Washbourne says. He plans to investigate the function of the forebrain social cells, and others, in fish with mutations in genes linked to autism.

In doing so, Washbourne expects to face political hurdles on top of scientific ones. Ask any autism researcher who works with zebrafish, flies or roundworms if their choice of model organism has ever raised an eyebrow, and they are likely to laugh knowingly. About 10 years ago, Washbourne submitted a grant application to the U.S. National Institutes of Health in which he proposed using zebrafish to study how neuronal junctions form. He remembers one reviewer asking, "Haven't people [already] done this in other invertebrates?" (Fish, of course, are vertebrates.) Those comments echo the resistance Rand encountered when trying to publish his worm work. 


\section{Spectrum | Autism Research News}

https://www.spectrumnews.org

But some researchers say the opposition is starting to wane. McDiarmid says he has noticed a shift in attitude since he joined Rankin's lab in 2014. More scientists are becoming attuned to the need for speed in certain situations, and that these alternative models can fill that need: "You can get a fast answer to a discovery question," McDiarmid says. "And it's not to replace mice at all; it's to prioritize [what to focus on]. I think that's the big concept shift."

Cite this article: https://doi.org/10.53053/QYKX3135 